With 1 plate

Printed in Great Britain

\title{
The Intracellular Location of the Agent of Mouse Scrapie
}

\author{
By G. D. HUNTER AND G. C. MILLSON \\ Agricultural Research Council, Institute for Research on \\ Animal Diseases, Compton, Newbury, Berks \\ AND G. MEEK \\ Department of Human Anatomy, University of Oxford
}

(Received 29 August 1963)

\begin{abstract}
SUMMARY
Homogenates of brains from mice clinically affected with scrapie have been fractionated by differential centrifugation and equilibration in sucrose density gradients. Most of the infectivity was found in the heavy particulate fraction containing mitochondria and possibly lysosomes.
\end{abstract}

\section{INTRODUCTION}

Scrapie is a chronic, progressive, degenerative disease of the central nervous system occurring naturally in sheep, and possibly in goats, and almost invariably terminating fatally. It can be transmitted from sheep to goats, and most of the experimental work carried out on the disease over the last 30 years has made use of these two large domestic animals (see reviews by Gordon, 1957, and Stamp, 1962). Recently a form of encephalopathy (termed mouse scrapie throughout this paper) has been produced in mice by inoculating them with brain material from scrapie-affected goats (Chandler, 1961, 1963), and it is now possible to make quantitative studies of the disease (Hunter, Millson \& Chandler, 1963) by carrying out titrations in large numbers of animals. Chandler's (1961) initial experiments have been successfully repeated in other laboratories (Morris \& Gajdusek, 1963; Eklund, Hadlow \& Kennedy, 1963; Zlotnik \& Rennie, 1963). Two groups of workers have further shown that it is possible to transmit the disease directly to mice from sheep (Morris \& Gajdusek, 1963; Zlotnik \& Rennie, 1963).

Although the scrapie agent has been characterized for some time as a filterpassing agent, a fact confirmed recently in the case of mouse scrapie (Chandler, 1963; Eklund et al. 1963), its exceptional resistance to chemical and physical agents has aroused much speculation as to its precise nature. Our earlier results (Hunter et al. 1963; Hunter, 1963) demonstrated the association of the agent of mouse scrapie with cytoplasmic particles. More refined methods of fractionation have now been applied to homogenates of brain from scrapie-affected mice and the bulk of the infectivity has been found to reside in the purified mitochondrial fraction of the cell. This fractionation may also be rich in lysosomes. Some of these results have been reported previously in a preliminary form (Hunter \& Millson, 1963). 


\section{METHODS}

Mice. B.S.V.S. (bacteria susceptible, virus susceptible, Schneider, 1959) white mice were used. Cases of scrapie were diagnosed as described previously (Hunter et al. 1963), following the criteria laid down by Chandler (1963) and by Pattison \& Smith (1963).

Cellular fractionation of mouse-scrapie brain. Four scrapie-affected mice were decapitated, their brains removed immediately and homogenized in a Potter-type homogenizer in $0.25 \mathrm{M}$-sucrose $\left(\mathbf{1 0 . 8 \mathrm { ml }}\right.$.) containing $5 \mathrm{mM}^{-\mathrm{CaCl}_{2}}$. A portion of the homogenate $(0.5 \mathrm{ml}$.) was set aside and used for the preparation of the saline dilutions of control material. The remaining material $(R)$ was maintained between $0^{\circ}$ and $4^{\circ}$ in all subsequent manipulations.

One half of the remaining material $(R)$ was transferred to a $3 \times 1$ in. lusteroid tube containing $0.88 \mathrm{M}$-sucrose $(10 \mathrm{ml}$.) covered with a separate layer of $0.32 \mathrm{M}$-sucrose $(6 \mathrm{ml}$.$) . After centrifugation at 53,500 \mathrm{~g}$ in a Spinco Model L SW. 25 rotor for $1 \mathrm{hr}$ the tube was placed in a dry ice-acetone mixture and the rapidly frozen 'top layer' sawn off with a small hack-saw. This fraction was allowed to thaw slowly in a small beaker, and the whole process repeated before saline dilutions were prepared from it for titration. As the material tended to adhere to the surface of glassware, saline dilutions for titration were prepared by using as far as was conveniently possible the whole of the material, and a pipette $(10 \mathrm{ml}$.) was used only when the volume to be handled exceeded 11 .

The other half of the homogenate $(R)$ was carefully pipetted on to the surface of $0.88 \mathrm{M}$-sucrose $(15 \mathrm{ml}$.) and centrifuged as above. The clear supernatant layer (soluble fraction $S$ ) was removed, transferred to a sterile tube and left at $2^{\circ}$ until required. The myelin layer $(M)$ at the interface between the 0.88 and $0.25 \mathrm{M}$-sucrose layers was removed as completely as possible and also transferred to a sterile tube at $2^{\circ}$. The bulk of the $0.88 \mathrm{M}$-sucrose solution $(88 \mathrm{~S})$ was now removed with minimal contamination with particulate matter, leaving a deposit containing nuclei, cell debris, mitochondria, etc. Distilled water (5.25 ml.) and 0.32 M-sucrose (3 ml.) were added to this deposit which was then gently rehomogenized before adding to $2 \mathrm{M}$ sucrose $(1 \mathrm{ml}$.) in a $3 \times 1$ in. lusteroid tube. The homogenizer was rinsed with $0.32 \mathrm{M}$ sucrose $(5 \mathrm{ml}$.) and the tube then centrifuged at $1000 \mathrm{~g}$ for $10 \mathrm{~min}$. The supernatant solution (NS 1) was removed carefully and the sedimented nuclear fraction resuspended in water $(5.25 \mathrm{ml}$.) and $0.32 \mathrm{M}$-sucrose $(3 \mathrm{ml}$.) and treated as before. After centrifuging the supernatant solution (NS 2) was combined with the previously obtained solution (NS 1). The nuclear deposit was resuspended in water $(5.25 \mathrm{ml}$.) and spread carefully on the surface of $0.88 \mathrm{M}$-sucrose $(15 \mathrm{ml}$.) in a $3 \times 1 \mathrm{in}$. lusteroid tube. The nuclear fraction was collected after centrifuging at $17,000 \mathrm{~g}$ for $55 \mathrm{~min}$. to remove most of the residual myelin. Dilutions in saline were prepared for titration in the usual way (Hunter et al. 1963) after a final sedimentation of the nuclei from $0 \cdot 32 \mathrm{M}$-sucrose at $1000 \mathrm{~g}$ for $10 \mathrm{~min}$.

The combined supernatant solutions (NS 1 and NS 2) removed from above the nuclear sediments were centrifuged at $17,000 \mathrm{~g}$ for $55 \mathrm{~min}$. and the supernatant solution now obtained (MS) retained at $2^{\circ}$ for the preparation of microsomal fractions. The deposit was resuspended in $0.32 \mathrm{M}$-sucrose and lightly homogenized before layering on to a discontinuous sucrose gradient constructed from $1.5 \mathrm{M}$-sucrose 
(0.5 ml.), 1.2 M-sucrose (10 ml.) and 0.88 M-sucrose (10 ml.). After centrifuging at $53,500 \mathrm{~g}$ for $2 \mathrm{hr}$ in a Spinco SW. 25 rotor, mitochondria were removed carefully from the 1.5-1.2M-sucrose interface, and nerve-ending particles from the 1.2$0.88 \mathrm{M}$ interface. The process was repeated on the separated fractions in order to effect further purification and they were finally sedimented into firm pellets $(105,000 \mathrm{~g}$ for $1 \mathrm{hr}$ ) from $0 \cdot 32 \mathrm{M}$-sucrose preparatory to the making of saline dilutions for titration as above.

The myelin fraction (M) obtained as above was layered on to the surface of $0 \cdot 88 \mathrm{M}$ sucrose $(20 \mathrm{ml}$.) and centrifuged at 53,500 $\mathrm{g}$ in a Spinco SW. 25 rotor for $2 \mathrm{hr}$. The myelin from the interface was collected in the minimum volume of liquid (approx. $2 \mathrm{ml}$.) and the suspension diluted with $0.32 \mathrm{M}$-sucrose $(10 \mathrm{ml}$.). It was finally resedimented at $105,000 \mathrm{~g}$ for $1 \mathrm{hr}$. Dilutions in saline for titration were prepared as above.

The clear supernatant layer (S) obtained from the first centrifugal run was recentrifuged at $105,000 \mathrm{~g}$ for $90 \mathrm{~min}$. before freezing rapidly in acetone-solid $\mathrm{CO}_{2}$. The frozen upper and lower portions of the solution were removed with a hack-saw, and the central portion (soluble fraction or cell sap) collected and thawed out before preparing saline dilutions for titration in the usual way. In this instance, all dilutions from $10^{-3}$ downwards were prepared directly from the sucrose solution isolated as above, but the $10^{-2}$ dilution was dialysed against saline before inoculating the mice as the sucrose concentration was rather high for intracerebral injection.

For the preparation of the microsomal fractions, the original $0.88 \mathrm{M}$-sucrose solution ( $88 \mathrm{~S}$ ) obtained above was first diluted to $0.32 \mathrm{M}$-sucrose and centrifuged at $10,000 \mathrm{~g}$ for $10 \mathrm{~min}$. The supernatant solution was combined with mitochondrial supernatant solution (MS) and centrifuged at $105,000 \mathrm{~g}$ for $90 \mathrm{~min}$. The supernatant solution was decanted and the microsomal pellet resuspended in $\mathbf{0 . 3 2} \mathrm{M}$ sucrose (1 ml.) and lightly rehomogenized. It was then layered on to a discontinuous sucrose gradient made up from 1.6 M-sucrose ( $1 \mathrm{ml}$.), 1.2 M-sucrose (1 ml.) and 1.0 Msucrose $(1 \mathrm{ml}$.). After centrifuging at $125,000 \mathrm{~g}$ for $1 \mathrm{hr}$ in a Spinco SW. 39 rotor three fractions were collected: (1) a 'debris' fraction, partly myelin, resting on the 0.32-1.0 M interface; (2) microsomes 1 at the 1.0-1.2M-sucrose interface; and (3) microsomes 2 at the $1 \cdot 2-1 \cdot 6 \mathrm{M}$-sucrose interface. The fractions were carefully removed, diluted to approximately $0.32 \mathrm{M}$-sucrose and sedimented at $105,000 \mathrm{~g}$ for $90 \mathrm{~min}$. Saline dilutions of each fraction were prepared for titration in the usual way. In every case, allowance was made for operational losses before preparing the dilutions.

The whole procedure adopted for the cellular fractionation is summarized in Scheme 1.

\section{Electron microscopy}

Samples of control and infected mitochondria and of infected microsomes were prepared as described above and were fixed in buffered osmium tetroxide solution (Palade, 1952) for $45 \mathrm{~min}$. The material was then centrifuged into pellets, dehydrated in a conventional ethyl alcohol series followed by propylene oxide and embedded in British Ciba Araldite. Sections were cut at about $60 \mathrm{~m} \mu$ on a Huxley ultramicrotome and examined in a Siemens Elmiskop I electron microscope. Micrographs were made at plate magnifications of around 20,000 using the double condenser system at $60 \mathrm{kV}$. 
Scheme 1. Subcellular fractionation of scrapie mouse brain by differential centrifugation and equilibration on discontinuous sucrose density gradients

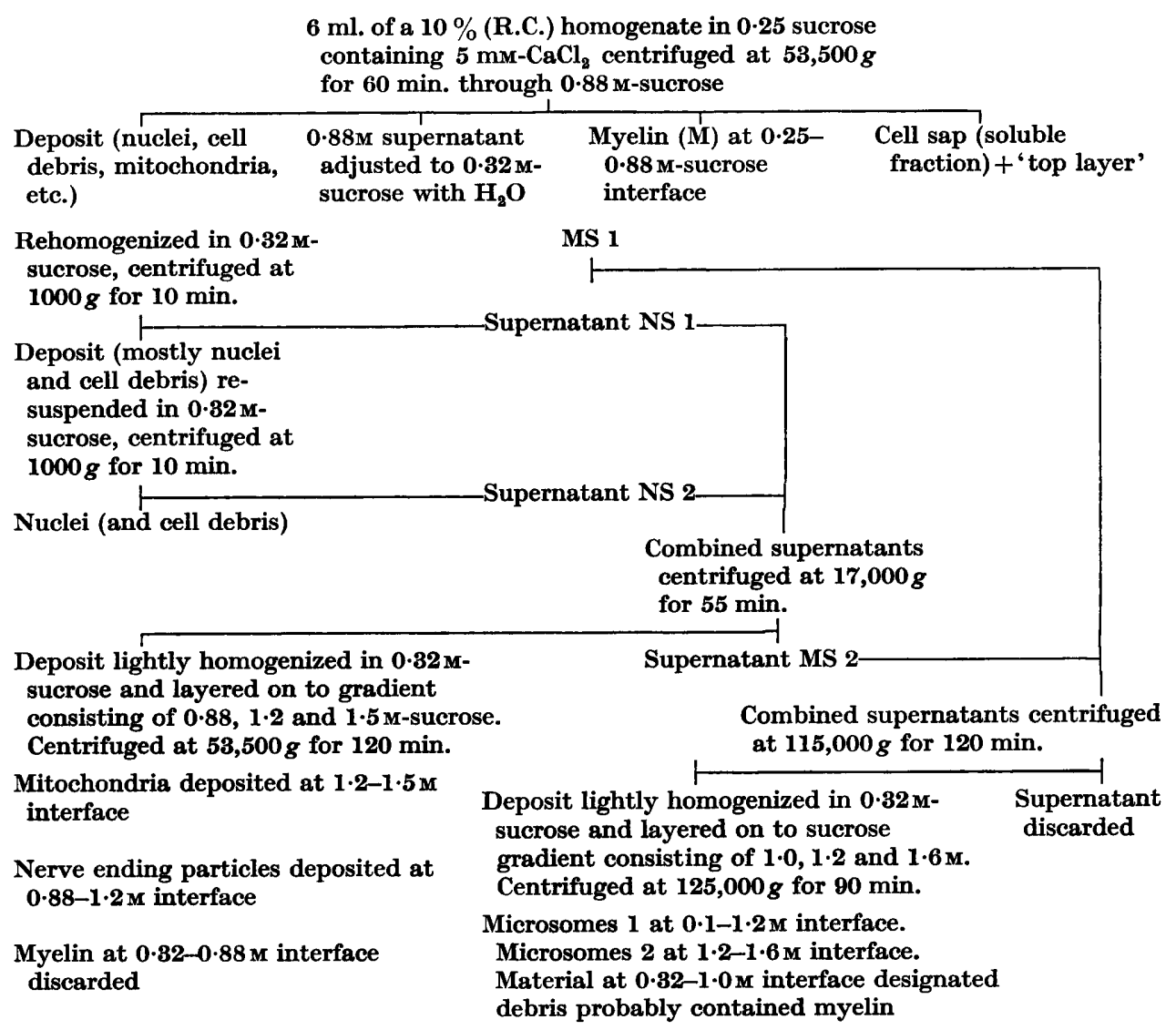

\section{RESULTS}

\section{Structure of cellular fractions}

The composition of the various cellular fractions was checked by electron microscopy. An electron micrograph of myelin prepared as described here has been shown previously (Hunter et al. 1963). Plate 1, figs. 1 and 2, show that the microsome fractions obtained here are fairly typical, and that only the microsome fraction 2 seems to contain appreciable numbers of free ribosomes. Figure 3 shows the mitochondrial fraction, which in addition to large numbers of mitochondria also contains other bodies of comparable size, possibly lysosomes. No particles resembling a large virus have been seen in any of the sections examined so far. Figure 4 shows a normal mouse brain mitochondrial preparation.

The only unusual feature arising from the cellular fractionation of the scrapie material is the appearance of a thin 'top layer' that floats on the top of the original homogenate after high-speed centrifugation. Electron microscopy showed that this fraction, which constituted only a very small proportion of the original homo- 
genate, consisted largely of myelin fragments and large osmiophilic granules that were almost certainly globules of fat.

\section{Potencies of the various cellular fractions}

The only material to approach the infected whole homogenate in potency was the mitochondrial fraction (Tables 1 and 2). In view of the large numbers of mice required, several of the fractions were assayed using a limited titration only (Hunter et al. 1963), and it is gratifying to see (Table 2) that the calculated infectivity of the mitochondrial fraction is practically the same using either method of assay. The myelin, nuclei and nerve ending particles also contained some scrapie agent, and the microsome fractions possibly slightly less. However, the 'top layer' and soluble fractions of the cell had very low infectivity.

Table 1. Percentage deaths from scrapie in groups of mice inoculated with various dilutions of purified cellular fractions

B.S.V.S. mice (groups of 8 ) were inoculated intracerebrally with materials prepared as described in the text. Clinical diagnosis and histopathological confirmation of the presence of mouse scrapie were carried out as described in Methods. The experiment was terminated 7 months after the inoculation.

Dilution of the inoculum related to whole brain

\begin{tabular}{lrrrrrrrrr}
\multicolumn{1}{c}{ Inoculum } & \multicolumn{1}{c}{ 10 $^{-2}$} & $10^{-8}$ & $10^{-4}$ & $10^{-5}$ & $10^{-6}$ & $10^{-7}$ & $10^{-8}$ & $10^{-9}$ & ID 50 \\
Control & 100 & 100 & 100 & 100 & 87 & 0 & 0 & 0 & $6 \cdot 4$ \\
Mitochondria & 100 & 100 & 100 & 100 & 71 & 0 & 0 & 0 & $6 \cdot 2$ \\
Microsomes 1 & 100 & 100 & 100 & 43 & 37 & 0 & 0 & 0 & $5 \cdot 3$ \\
Microsomes 2 & 100 & 100 & 75 & 25 & 12 & 0 & 0 & 0 & $4 \cdot 6$ \\
'Top layer' & 75 & 25 & 0 & 0 & 0 & 0 & 0 & 0 & $2 \cdot 5$ \\
Soluble & 100 & 100 & 0 & 0 & 0 & 0 & 0 & 0 & $\mathbf{3} \cdot 5$
\end{tabular}

Table 2. Incubation periods of scrapie in groups of mice inoculated with various dilutions of purified cellular fractions

The data refer to the same experiment as that described in Table 1. The calculations of infectivity based on length of the incubation period are made according to Hunter et al. (1963), who defined ST 50 for each group as the time after inoculation by which $50 \%$ of the mice in the group had been killed in the final clinical stage of the disease.

\begin{tabular}{|c|c|c|c|c|c|}
\hline \multirow[b]{2}{*}{ Inoculum } & \multicolumn{3}{|c|}{ ST 50 in days of dilution group } & \multirow{2}{*}{$\begin{array}{c}\% \text { of control } \\
\text { infectivity } \\
\text { based on } \\
\text { length of } \\
\text { incubation } \\
\text { periods }\end{array}$} & \multirow{2}{*}{$\begin{array}{c}\% \text { of control } \\
\text { infectivity } \\
\text { based on } \\
\text { complete } \\
\text { titration }\end{array}$} \\
\hline & $10^{-2}$ & $10^{-8}$ & $10^{-4}$ & & \\
\hline Control & 136 & 146 & 146 & 100 & 100 \\
\hline Myelin & 154 & 154 & 171 & 13 & - \\
\hline Nuclei & 146 & 154 & 164 & 23 & - \\
\hline Mitochondria & 136 & 136 & 168 & 62 & 63 \\
\hline Nerve ending particles & 136 & 146 & 182 & 23 & - \\
\hline Microsomes 1 & 139 & 157 & 189 & 10 & $7 \cdot 9$ \\
\hline Microsomes 2 & 146 & 154 & 182 & 11 & $1 \cdot 6$ \\
\hline 'Debris' & 164 & 202 & - & - & - \\
\hline 'Top layer' & 189 & 一 & - & - & 0.013 \\
\hline Soluble & 168 & 182 & - & - & $0 \cdot 13$ \\
\hline
\end{tabular}




\section{DISCUSSION}

The method of cellular fractionation used here is based to some extent on that used by Gray \& Whittaker (1962) who were mainly concerned with the preparation of nerve-ending particles from guinea-pig brain. The only unusual feature, the obtaining of a 'top layer' when centrifuging scrapie homogenates, does not appear to be of any fundamental importance. The 'top layer' contains very little of the infective agent and probably consists of myelin fragments and cytoplasmic fat globules arising from degenerate cells. Otherwise the electron micrographs show that all the fractions examined closely resembled those obtainable from normal brain homogenates. The mitochondrial fraction contained a number of bodies that could possibly be lysosomes.

The high infectivity found in the mitochondrial fraction, incidentally representing a five- to tenfold concentration of the agent, would at first sight suggest that the scrapie agent is a large particle with a size and density somewhat similar to that of brain mitochondria. The finding of a considerable scrapie potency in the fractions most similar in size and density to the mitochondria (i.e. nuclei and nerve ending particles) would be consistent with this suggestion, and these two fractions would contain some contaminating mitochondria. However, we have been unable to find any object resembling a large virus in any of the electron micrographs, and it is also somewhat surprising to find an appreciable, if low, titre in the soluble fraction if a large virus is concerned.

A second possibility may be that the agent is a small or medium-sized virus which is for some reason specifically adsorbed to mitochondria or lysosomes, or, perhaps, multiplies within these larger organelles. If the lysosomes were the organelles concerned, the lytic action of scrapie upon brain cells would be readily understandable. Eklund et al. (1963) consider that the scrapie agent is a medium-sized virus largely on the basis of filtration evidence. It is not clear, however, what proportion of the infectivity actually passed through a $100 \mathrm{~m} \mu$ filter. The filtered material in their hands was no longer resistant to boiling and it seems possible that their filter removed all the mitochondrial-bound agent.

In either case, an experimental attack involving disruption of the large mitochondrial organelles by physical and chemical agents should provide valuable information. Transfer of the bulk of the residual infectivity to the microsomal fractions would then indicate the presence of a small mitochondrial- or lysosomalbound agent. On the other hand, if the bulk of the residual infectivity could be transferred to the soluble fraction of the cell, it would appear that scrapie involves a derangement in protein metabolism, the presence of a colicin-type factor, or the introduction of a toxic protein capable of stimulating its own reproduction.

Myelin, nuclear and mitochondrial fractions obtained here retained higher absolute potencies than the corresponding fractions prepared previously from scrapie brain (Hunter et al. 1963) by a rather less elaborate density gradient procedure. The scrapie agent appears to be stable in strong solutions of sucrose (Hunter \& Millson, 1963), but it is clear that in certain circumstances, at present not precisely understood, cellular fractionation can lead to a loss of scrapie infectivity. 
Journal of General Microbiology, Vol. 34, No. 2

Plate 1



Fig. 1



Fig. 3

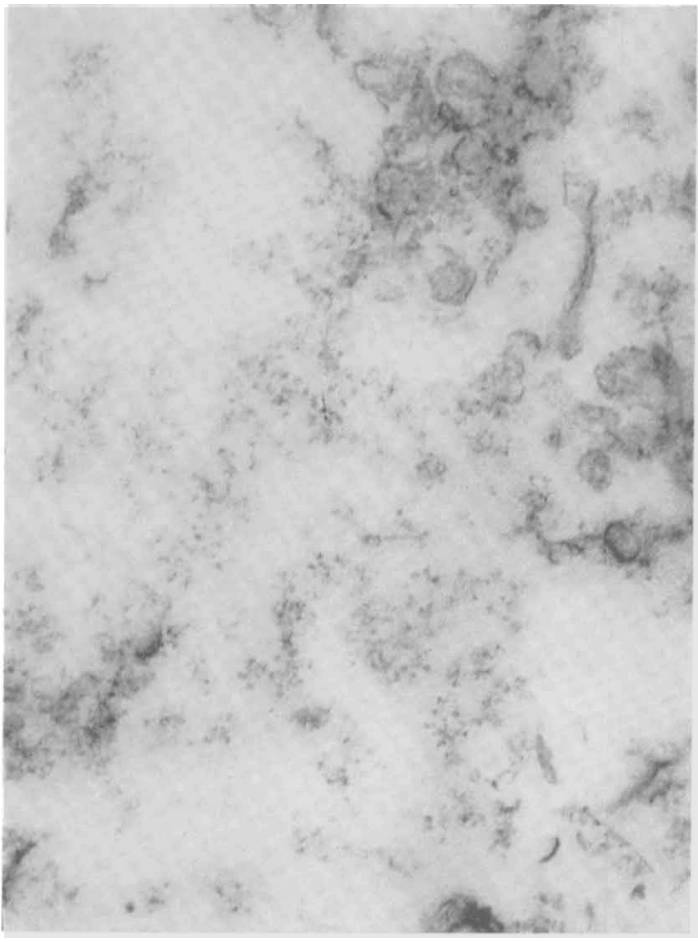

Fig. 2

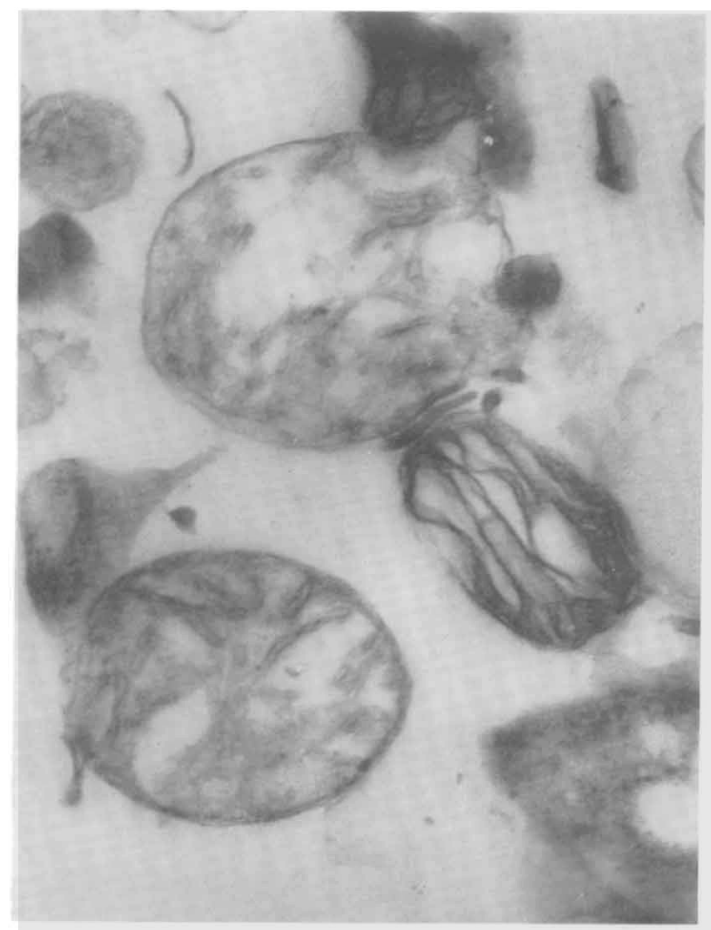

Fig. 4 
The authors are grateful to Dr W. S. Gordon, C.B.E., for his continued interest and advice, and to Dr A. Mackenzie and Mr P. Dennis for preparing the histological specimens. They also wish to acknowledge the skilled technical assistance of $\mathbf{M r} \mathbf{M}$. Vockins, and the excellent work carried out by the staff of the animal house in the breeding and handling of mice. This work was supported in part by grants made to the A.R.C. from U.S. Government funds administered under U.S. Public Law 480.

\section{REFERENCES}

Chandler, R. L. (1961). Encephalopathy in mice produced with scrapie brain material. Lancet, $\mathrm{i}, 1378$.

Chandler, R. L. (1963). Experimental scrapie in the mouse. Res. vet. Sci. 4, 276.

Eklund, C. M., Hadlow, W. J. \& Kennedy, R. C. (1963). Some properties of the scrapie agent and its behaviour in mice. Proc. Soc. exp. Biol., N.Y. 112, 974.

Gordon, W. S. (1957). Studies in scrapie. Vet. Rec. 69, 1324.

Gray, E. G. \& WhitTaker, V. P. (1962). The isolation of nerve endings from the brain: an electron-microscopic study of cell fragments derived by homogenisation and centrifugation. J. Anat. 96, 79.

HUNTER, G. D. (1963). Biochemical investigations on the nature of the agent of 'mousescrapie'. J. gen. Microbiol. 31, xv.

HunTer, G. D. \& Mrluson, G. C. (1963). The comparative infectivities of extracts of tissues and of purified cell fractions from mice infected with the scrapie agent. Biochem. $J$. 89, $42 P$.

Hunter, G. D., Mrllson, G. C. \& Chandrer, R. L. (1963). Observations on the comparative infectivity of cellular fractions derived from homogenates of mouse-scrapie brain. Res. vet. Sci. (in the Press).

MorRIs, J. A. \& GAJDUSEK, D. C. (1963). Encephalopathy in mice following inoculation of scrapie sheep brain. Nature, Lond. 197, 1084.

Palade, G. E. (1952). A study of fixation for electron microscopy. J. exp. Med. 95, 285.

Pattison, I. M. \& Smith, K. (1963). Histological observations on experimental scrapie in the mouse. Res. vet. Sci. 4, 269.

SchNeIDER, H. A. (1959). Genetic and nutritional aspects of experimental 'allergic' encephalomyelitis, in 'Allergic' Encephalomyelitis, ch. 3, p. 130. Ed. by M. W. Kies and E. C. Alvord. Oxford: Blackwell Scientific Publications, Ltd.

Stamp, J. T. (1962). Scrapie; a transmissible disease of sheep. Vet. Rec. 74, 357.

Zlotnik, I. \& RenNie, J. C. (1963). Further observations on the experimental transmission of scrapie from sheep and goats to laboratory mice. J. comp. Path. 73, 150.

\section{EXPLANATION OF PLATE}

Fig. 1. Electron micrograph of microsomes 1 fraction prepared as described in the text (magnification $\times \mathbf{5 3 , 0 0 0 )}$.

Fig. 2. Electron micrograph of microsomes 2 fraction prepared as described in the text (magnification $\times \mathbf{5 3 , 0 0 0 )}$.

Fig. 3. Electron micrograph of mitochondria prepared from scrapie mouse brain as described in the text (magnification $\times \mathbf{5 3 , 0 0 0 )}$.

Fig. 4. Electron micrograph of normal mouse brain mitochondria (magnification $\times 53,000$ ). 